Supplement of Biogeosciences, 13, 13-26, 2016

http://www.biogeosciences.net/13/13/2016/

doi:10.5194/bg-13-13-2016-supplement

(C) Author(s) 2016. CC Attribution 3.0 License.

(c) (i)

Supplement of

\title{
Phototrophic pigment diversity and picophytoplankton in permafrost thaw lakes
}

\section{A. Przytulska et al.}

Correspondence to: A. Przytulska (anna.przytulska.1@ulaval.ca)

The copyright of individual parts of the supplement might differ from the CC-BY 3.0 licence. 


\section{Supplementary material}

Table S1. Location (latitude and longitude), maximum depth $(\mathrm{Z})$ of the subarctic lakes and sampling dates. Water was sampled ca. $20 \mathrm{~cm}$ below the surface and ca. $20 \mathrm{~cm}$ above the maximum depth. The shallow rock basin lakes have been referred elsewhere as follows: WP1 (SRB1), WP2 (SRB2), Olsha (SRB3), 4 KM (SRB4), Iqalusiuvik (SRB5), - no sampling.

\begin{tabular}{|c|c|c|c|c|c|}
\hline \multirow[t]{2}{*}{ Lakes } & \multirow[t]{2}{*}{ Latitude } & \multirow[t]{2}{*}{ Longitude } & \multirow{2}{*}{$\begin{array}{c}\mathrm{Z} \\
(\mathrm{m})\end{array}$} & \multicolumn{2}{|c|}{ Sampling dates } \\
\hline & & & & 2011 & 2012 \\
\hline \multicolumn{6}{|c|}{ Thaw lakes on northern clay soils } \\
\hline BGR1 & $56^{\circ} 36.6500^{\prime} \mathrm{N}$ & $76^{\circ} 12.900^{\prime} \mathrm{W}$ & 3.5 & $20 \mathrm{Aug}$ & 9 Aug \\
\hline BGR2 & $56^{\circ} 36.632^{\prime} \mathrm{N}$ & $76^{\circ} 12.937^{\prime} \mathrm{W}$ & 1.0 & 20 Aug & 9 Aug \\
\hline NASA & $56^{\circ} 55.434{ }^{\prime} \mathrm{N}$ & $76^{\circ} 22.708^{\prime} \mathrm{W}$ & 3.2 & 7 Aug & - \\
\hline NASH & $56^{\circ} 55.452^{\prime} \mathrm{N}$ & $76^{\circ} 22.636^{\prime} \mathrm{W}$ & 3.6 & 7 Aug & - \\
\hline \multicolumn{6}{|c|}{ Thaw lakes on southern clay soils } \\
\hline KWK1 & $55^{\circ} 19.8900^{\prime} \mathrm{N}$ & $77^{\circ} 30.241^{\prime} \mathrm{W}$ & 2.1 & 19 Aug & $3 \mathrm{Aug}$ \\
\hline KWK6 & $55^{\circ} 19.937 ' \mathrm{~N}$ & $77^{\circ} 30.117^{\prime} \mathrm{W}$ & 3.2 & 21 Aug & 4 Aug \\
\hline KWK12 & $55^{\circ} 19.808^{\prime} \mathrm{N}$ & $77^{\circ} 30.239^{\prime} \mathrm{W}$ & 2.6 & 19 Aug & 3 Aug \\
\hline KWK23 & $55^{\circ} 19.947{ }^{\prime} \mathrm{N}$ & $77^{\circ} 30.131^{\prime} \mathrm{W}$ & 3.4 & 21 Aug & 4 Aug \\
\hline \multicolumn{6}{|c|}{ Thaw lakes on peatlands } \\
\hline SAS1A & $55^{\circ} 13.128^{\prime} \mathrm{N}$ & $77^{\circ} 42.477^{\prime} \mathrm{W}$ & 1.9 & 23 Aug & 5 Aug \\
\hline SAS1B & $55^{\circ} 13.143^{\prime} \mathrm{N}$ & $77^{\circ} 42.475^{\prime} \mathrm{W}$ & 1.7 & 23 Aug & 5 Aug \\
\hline SAS2A & $55^{\circ} 13.591{ }^{\prime} \mathrm{N}$ & $77^{\circ} 41.815^{\prime} \mathrm{W}$ & 2.6 & - & 13 Aug \\
\hline SAS2B & $55^{\circ} 13.600^{\prime} \mathrm{N}$ & $77^{\circ} 41.806^{\prime} \mathrm{W}$ & 2.0 & - & 13 Aug \\
\hline \multicolumn{6}{|c|}{ Shallow rock-basin lakes } \\
\hline SRB1 & $55^{\circ} 16.982^{\prime} \mathrm{N}$ & $77^{\circ} 44.187^{\prime} \mathrm{W}$ & 0.4 & 24 Aug & 11 Aug \\
\hline SRB2 & $55^{\circ} 16.970{ }^{\prime} \mathrm{N}$ & $77^{\circ} 44.122^{\prime} \mathrm{W}$ & 0.8 & 24 Aug & 11 Aug \\
\hline SRB3 & $55^{\circ} 16.958^{\prime} \mathrm{N}$ & $77^{\circ} 44.387^{\prime} \mathrm{W}$ & 1.6 & $24 \mathrm{Aug}$ & 14 Aug \\
\hline SRB4 & $55^{\circ} 19.907 ’ \mathrm{~N}$ & $77^{\circ} 41.959^{\prime} \mathrm{W}$ & 0.7 & 16 Aug & 8 Aug \\
\hline SRB5 & $55^{\circ} 22.262^{\prime} \mathrm{N}$ & $77^{\circ} 37.072^{\prime} \mathrm{W}$ & 1.8 & $12 \mathrm{Aug}$ & 8 Aug \\
\hline
\end{tabular}

